\title{
Seeing ahead: Experience and language in spatial perspective
}

\author{
TRACY PACKIAM ALLOWAY \\ University of Durham, Durham, England \\ MARTIN CORLEY \\ University of Edinburgh, Edinburgh, Scotland \\ and \\ MICHAEL RAMSCAR \\ Stanford University, Stanford, California
}

\begin{abstract}
Spatial perspective can be directed by various reference frames, as well as by the direction of motion. In the present study, we explored how ambiguity in spatial tasks can be resolved. Participants were presented with virtual reality environments in order to stimulate a spatial reference frame based on motion. They interacted with an ego-moving spatial system in Experiment 1 and an object-moving spatial system in Experiment 2. While interacting with the virtual environment, the participants were presented with either a question representing a motion system different from that of the virtual environment or a nonspatial question relating to physical features of the virtual environment. They then performed the target task: assign the label front in an ambiguous spatial task. The findings indicate that the disambiguation of spatial terms can be influenced by embodied experiences, as represented by the virtual environment, as well as by linguistic context.
\end{abstract}

Spatial perspective can be understood through a variety of reference frames, such as intrinsic, relative, and absolute relations (Levinson, 1996). In an intrinsic reference frame, the inherent spatial properties of an object determine spatial perspective. In a relative reference frame, an object is judged as abovelbelow or front/behind on the basis of the viewer's perspective, regardless of its inherent spatial properties. An absolute reference frame is one that refers to an object's location with respect to external surroundings, such as those provided by gravity or cardinal directions. These different ways of understanding spatial perspective are evident across languages (Couclelis, Golledge, Gale, \& Tobler, 1987).

The use of spatial terms when a person is described is directed primarily by an intrinsic reference scheme. For example, the top is taken to be the head of the person,

Some of the data in this article were reported at the 23rd Conference of the Cognitive Science Society. The research was conducted while the first author was in receipt of an Overseas Research Student Award from the committee of Vice-Chancellors and Principals of the Universities of the United Kingdom and a Postgraduate Research Studentship from the Faculty Group of Law and Social Sciences, University of Edinburgh, and forms part of her doctoral thesis at the University of Edinburgh. The authors thank Jon Sykes for his assistance in programming the virtual reality environments used in the experiments. We also extend our gratitude to Simon Garrod, Rolf Zwaan, and two anonymous reviewers for helpful comments on an earlier draft. Correspondence concerning this article should be addressed to T. P. Alloway, Department of Psychology, University of Durham, Science Laboratories, South Road, Durham DH1 3LE, England (e-mail: t.p.alloway@durham.ac.uk). and the bottom is taken to be the feet. Left and right correspond to the labels assigned to the left and right sides of the human body; the front is associated with the orientation of the perceptual organs (i.e., the eyes), and the back is the opposite location (see Fillmore, 1971; Miller \& JohnsonLaird, 1976). This reference scheme is also generalizable to animals and objects on the basis of a coincidence or an encounter situation (Herskovits, 1986). In the coincidence situation, for an animal, a spatial orientation is adopted that is similar to the intrinsic assignments of front/behind, top/bottom, and left/right to a person. It is important to note, however, that the intrinsic reference frame for people is not directly mapped onto animals. Instead, spatial assignments correspond to an animal's natural physical orientation. For example, the front of a dog is not its stomach, as it is for people, but the front of its head.

Objects can also be given similar spatial assignments. In a coincidence situation, the front of an object is thought to be the front in its natural orientation. In an encounter situation (Herskovits, 1986), spatial terms are assigned relative to the viewer's perspective. However, the assignment of spatial relations to inanimate objects is complicated by a third possible reference frame: the accidental intrinsic scheme. In this scheme, symmetrical objects take on the spatial features unique to the environment that they are placed or occur in. For example, an object such as a tree will adopt spatial perspectives of front and back of a street; the front of the tree is the part encountered by the viewer, whereas the back is the side unseen from the viewer's perspective from the street (Fillmore, 1982). 
There is evidence suggesting that when people use spatial terms such as front and back, they are influenced more by intrinsic or relative reference frames than by absolute ones. For example, developmental research has indicated that young children assign intrinsic spatial labels to an object when it has an inherent front and back (e.g., Harris \& Strommen, 1972). Adult research also indicates that the inherent characteristics of an object, spatial coordination between the speaker and the listener, and the perspective of the scene will be more likely to influence the choice of reference frames that an individual adopts, as compared with absolute references, such as cardinal directions (Carlson-Radvansky \& Logan, 1997).

Spatial perspective can also be understood via motion as represented by two distinct systems: object moving and ego moving (Boroditsky, 2000; Gentner \& Imai, 1992; McGlone \& Harding, 1998). In the object-moving motion system, the individual is stationary and observes objects come toward him-for example, while waiting at a bus stop. The individual assigns the term front to the object closest to him. In Figure 1, the term front is assigned to the white rock.

The ego-moving motion system is analogous to the encounter situation described above. People experience this system when walking on a street past shops, houses, and parked cars or in a room past tables and chairs. In this motion system, the term front is assigned to the object farthest away from the individual. In Figure 2, the black rock is labeled as front. Field studies have confirmed that when individuals experience a particular motion system, this can inform their spatial perspective (Boroditsky \& Ramscar, 2002). For example, people at airports and train stations who had just landed at a destination or were anticipating a journey were more likely to adopt an ego-moving perspective of motion.

In the present study, we investigated how ambiguous spatial terms are understood, focusing specifically on the role of experience. Some researchers have suggested that language - in particular, abstract or ambiguous words - is grounded in daily embodied experiences (Lakoff \& Johnson, 1999). This is consistent with research on embodied cognition, an area that highlights the influential role the environment plays in the development of cognitive structures (e.g., Barsalou, 1999; Glenberg, 1997). Although there are various extensions of this view, a common theme is that the sensorimotor capacities of an individual who is interacting with the environment play a key role in directing specific cognitive facilities.

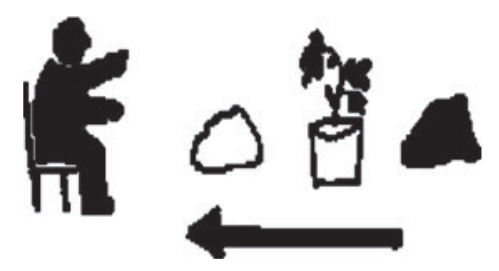

Figure 1. Schematic diagram representing the object-moving system of spatial perspective.

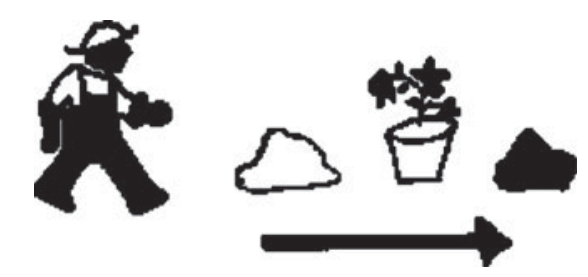

Figure 2. Schematic diagram representing the ego-moving system of spatial perspective.

The role of experience in spatial perspective was tested more directly by Alloway, Ramscar, and Corley (1999), using an interactive video game that adopted an objectmoving perspective. The participants who received an object-moving linguistic prime during the game were more likely to overcome the ego-moving bias of the target task and interpreted front consistently with the object-moving schema of the video game. In contrast, the participants who were interacting with the object-moving game but did not receive a linguistic prime were less likely to cross-map the representation of motion from the prime task to the target task. A priming check question indicated that the participants in both conditions were influenced by the objectmoving perspective of the game. The findings indicate that the embodied experience of motion was not sufficient to influence spatial perspective. However, linguistically representing the object-moving perspective boosted the benefits of the embodied experience.

Until recently, most studies in which the roles of language and experience in motion in space have been explored have been restricted to paper-and-pencil tests (e.g., Boroditsky, 2000) or two-dimensional video games (Alloway et al., 1999). However, in order to fully understand the role of experience while maintaining a controlled experimental environment, it is important to consider more interactive approaches to simulating motion. The decision to use a virtual environment to induce motion in the present study was based on the premise that perception is powerful enough to direct kinesthesis or movement (e.g., Sun, Chan, \& Campos, 2004; Waller, Loomis, \& Haun, 2004).

Another key aspect of previous studies on motion is the use of an ambiguous target task. Given the variety of spatial reference frames, it is unsurprising that spatial relationships and spatial terms can be ambiguous. For example, when hearing the statement that " $\mathrm{X}$ is in front," the listener has to first determine the speaker's spatial perspective before decoding the meaning of the phrase "in front." Boroditsky (2000) investigated the role of priming between the object-moving and the ego-moving motion perspectives for ambiguous spatial terms. The findings indicated that the participants were more likely to disambiguate a target question, such as "Next Wednesday's meeting has been moved forward two days," consistently with the system of motion they were exposed to. The participants who received ego-moving primes were more likely to answer the question as Friday, whereas those who saw object-moving primes responded that the meeting had been moved to Monday. 
In the present study, the participants had to respond to an ambiguous spatial task after interacting with a virtual environment in which they experienced either an egomoving or an object-moving spatial system, in Experiments 1 and 2, respectively. In order to test the roles of experience and language in directing spatial perspective, there were two experimental conditions. In one condition, half the participants received a nonspatial question relating to physical features of the environment. The remaining participants were presented with a question representing a different motion system than the virtual environment. For example, in Experiment 1, half the participants in the ego-moving virtual environment were presented with an object-moving question. After interacting with the virtual environment for a designated period, the participants then had to respond to an ambiguous spatial task. We expected that if spatial perspective is informed by an individual's immediate experience, he or she would be more likely to disambiguate the target task in a manner consistent with the motion system experienced in the virtual environment. For example, when experiencing an ego-moving system, the participant would be more likely to consider the front object as the one farthest away from him or her, in agreement with this spatial perspective. However, when experiencing an object-moving system, the participant would be more likely to consider the front object as the one closest to him or her, in agreement with this spatial perspective. However, if language can inform spatial perspective (e.g., Alloway et al., 1999), participants who received the linguistic prime while immersed in the virtual environment would respond differently to the target task than would those who were presented with the nonspatial question.

\section{EXPERIMENT 1}

\section{General Method}

\section{Participants}

Ninety-two students from the University of Edinburgh, the University of Durham, and various secondary schools in the U.K. participated in this experiment. Of these, 25 students were assigned to Baseline Condition 1, 31 to Baseline Condition 2, and the remainder to the experimental condition. Of the 36 participants in the experimental condition (mean age $=16 ; S D=0.65$ ), the demographic information from questionnaires showed that out of the 13 male and 23 female participants, only $3 \%$ played video games on a weekly basis. Since all the participants were familiar with using a computer, with $83 \%$ relying more on the arrow keys than on the computer mouse to navigate when typing, the use of arrow keys for navigating in the virtual environment did not raise problems for the participants.

\footnotetext{
Materials

In order to create a particularly convincing ego-moving environment, volunteers played a preexisting section of the virtual reality computer game Unreal, which was modified slightly for this experiment. The section of the game that was used consisted of a first-person perspective game and involved the volunteer's walking through a courtyard environment to complete a given task: "Find the location of a young woman." Close to the starting point of the virtual environment were two pillars positioned in a row: first, a green pillar and then a red one. These pillars were the focus of the linguistic prime question: "During the game, the green pillar is in front of the red pillar-True/ False." There were also doors on the buildings in the courtyard; some
}

were open, and others were closed. The doors were the objects of the nonspatial question: "During the game, most of the doors are open-True/False." The virtual environment was designed in such a manner as to encourage a forward trajectory of motion and was spacious enough that the participant would experience a different location if he or she moved forward continuously for 7-8 $\mathrm{min}$.

The only avatar present in the environment was the object of the task: the virtual woman designed specially for the experiment. She congratulated the participants upon successfully completing the task and then asked them to perform the target task: "Move to the front chest." Next to the virtual woman were two wooden chests, with no discernible front or back. The wooden chests were visible only once the participants had reached this point of the game. These were added from the Unreal directory of furniture in order to maintain continuity in the environment. The game was projected onto a $368 \mathrm{~cm}(12 \mathrm{ft}) \times 282 \mathrm{~cm}(9 \mathrm{ft})$ screen, $640 \times 480$ pixels.

\section{Baseline Condition 1}

\section{Procedure}

We investigated the ambiguity of assigning the term front to one of two wooden chests in the target task. Each participant was seated in front of the projector screen and was tested individually. The virtual game was set at the point in front of the two chests, which were positioned in a row (see Figure 3 ). The participants did not play game and were exposed to the virtual environment only for a brief period as they received instructions to "Move to the front chest," using the arrow keys on the computer keyboard.

\section{Results}

Twelve of the 25 participants (48\%) in the baseline condition interpreted the term front to refer to Chest $\mathrm{A}$, and the rest $(n=13)$ assigned front to Chest B. A chi-square analysis indicated that the difference between responses to the target task was not significant $\left[\chi^{2}(1)=0.04, p=\right.$ .84]. This finding indicates that there is an ambiguity in assigning the term front in the target task.

\section{Baseline Condition 2}

It is possible that the results from the first baseline condition could be confounded by introducing motion into the task (e.g., "Move to the front chest"). Thus, the second baseline condition addressed this possibility, using a nonmovement paradigm. The images were generated for this baseline condition.

\section{Procedure}

The participants were seated in front of a projector screen and were shown a PowerPoint slide with a gray and a red box positioned in a row, as illustrated in Figure 3. The gray box corresponded to Chest A, and the red box to Chest B. The participants had to identify
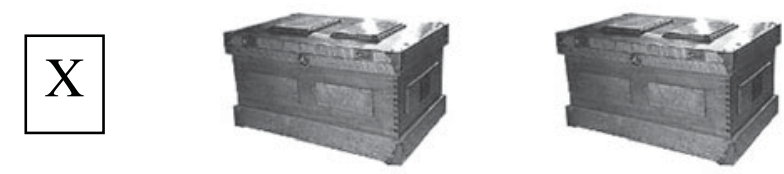

Participant

Chest A

Chest B

Figure 3. Position of wooden chests in the ego-moving virtual environment in relation to the participant. 
"Which is the front box: the gray one or the red one?" by circling the correct response on a questionnaire.

\section{Results}

Fourteen of the 31 participants $(45 \%)$ in the baseline condition interpreted the term front to refer to gray box, and the rest $(n=17)$ assigned front to the red one. A chisquare analysis indicated that there was not a significant difference in the assignment of the label front in the target task $\left[\chi^{2}(1)=0.29, p=.59\right]$. This finding confirms that the assignment of the label front in the target task is ambiguous even in a nonmovement condition.

\section{Experimental Condition}

\section{Procedure}

Each participant was tested individually on one trial. Before beginning the experiment, the participants were presented with some questions on demographics, as well as on their familiarity with computers and video games. Then they received the following instructions:

Your task is to find the location of a young woman. Try your best to navigate around the environment in order to find her. During this game, it is important to try and remember some key landmarks, such as a pair of brightly coloured pillars as you enter a path, as well as the doors on the buildings. After you have been playing for some time, you will hear a question requiring a true or false answer. This question will be about the game. Try to answer it correctly and speak your answer loudly.

The participants were led to the virtual reality game and were told to use the arrow keys on the keyboard when navigating through the environment. They were left on their own to play the game. The experimenter was on hand, however, should the volunteers have any difficulty maneuvering around the environment.

There were two experimental conditions. In the first condition, the participants received a prerecorded linguistic prime specific to the assignment of the term front approximately 4 min into playing the game. The linguistic prime was presented after a set amount of time, in order to guarantee that all the participants would have had equal exposure to the virtual environment. Some of the participants moved faster than others in the environment, and presenting the prime at a particular location would have meant that some of the participants would have had more exposure to the virtual environment than did others. Half of the participants had to respond true/ false to the following linguistic prime: "During the game, the green pillar is in front of the red pillar." The response consistent with the object-moving perspective of the prime was true. In order to counteract an affirmative response bias, the other half of the participants responded to the following linguistic prime: "During the game, the red pillar is in front of the green pillar." The response consistent with the object-moving perspective of the prime was false.

In the second condition, the participants received a prerecorded question that did not refer to the spatial properties of any of the objects in the environment. Approximately 4 min into playing the game, the participants had to answer true or false to the following question: "During the game, most of the doors are open." Half of the participants in this condition answered this question: "During the game, most of the doors are closed." The answer to this question depended on the route the participant chose when navigating around the environment to complete the task. Responses in this condition were not scored for correctness.

After receiving either a linguistic prime or a nonspatial question, the participants continued to navigate around the environment for a minimum of two more minutes. Once the participants had found the virtual young woman, she congratulated them and asked them to complete the target task, using the game console: "Move to the front chest." Upon completion of the target task, the participants were given a short debriefing.

\section{Results and Discussion}

Of the 18 participants who received the linguistic prime in the first experimental condition, 2 of them did not answer the prime question in a manner consistent with the object-moving perspective of the prime. These data were not used in the following analyses.

In the linguistic prime condition, all of the remaining 16 participants identified the green pillar as being located in front of the red pillar, regardless of the version of prime question. This means that all the participants who received the question "During the game, the green pillar is in front of the red pillar" responded true, and the participants who received the question "During the game, the red pillar is in front of the green pillar" responded false.

Of the participants who received the nonspatial question $(n=18), 83 \%$ of them selected the farther chest (Chest B in Figure 3), which is consistent with an egomoving perspective of the virtual environment. However, of the participants who received the linguistic prime, only $25 \%$ of them selected the farther chest. The majority of participants in the first condition (75\%) selected Chest A as the front chest, which is consistent with the object-moving perspective of the linguistic prime. A $2 \times 2$ chi-square analysis confirmed a significant difference in the interpretation of the term front in the ambiguous target task $\left[\chi^{2}(1)=11.7, p<.001\right]$.

The findings indicate that the participants were significantly influenced by the system of motion they represented. The participants who were presented with an objectmoving linguistic prime overcame the embodied ego-moving schema of motion when responding to the target task. In contrast, the participants who received a nonspatial question, unrelated to any system of motion, were influenced by the ego-moving schema in the virtual environment and responded to the target task accordingly.

\section{EXPERIMENT 2}

The first experiment required the participants to interact with an ego-moving virtual environment, in order to investigate how experience affects our understanding of ambiguous spatial labels. In the following experiment, the participants interacted with an object-moving environment and then responded to an ambiguous spatial target task.

\section{Method}

Participants. The participants were 30 students from the University of Edinburgh and various secondary schools in the U.K. (mean age $=18$ years, $S D=4.0$ ). The demographic information from the questionnaires revealed that out of the 14 males and 16 females, all of them used a computer on a regular basis, an advantage that allowed them to engage in the virtual environment without technical concerns (such as how to use the arrow keys). Furthermore, only $14 \%$ of the participants consistently played virtual reality games on a weekly basis, minimizing a familiarity bias of a virtual environment. None of the volunteers had participated in the previous experiment.

Materials. In order to create a realistic environment to represent an object-moving perspective, a new environment was designed using the tools and features from the same virtual reality game (Unreal) as that in the previous experiment. It was not appropriate 
to use the ego-moving layout for this experiment, since it included buildings and courtyards that potentially would have obscured the viewing of objects moving toward an individual. Furthermore, the layout for an object-moving perspective had to be relatively simple, with a straight view from the front to the back of the layout, in order to effectively portray objects moving toward an individual. This particular environment was an enclosed cave, with a doorway at the back, represented at the top middle of the screen. The participant was confined to one location, represented at the bottom middle of the screen. On the left side of the screen, there were two different types of trees, placed in a vertical line, similar to the position of the wooden chests in the previous experiment. On the right side of the screen, there was a large winged monster that remained there for the first 2-3 min of the game. It was the object of the linguistic prime: "During the game, the winged creature is in front of other creatures." From the doorway at the back of the environment, human-like creatures approached the participant, in order to represent an objectmoving environment.

The target task in this environment was to "Identify the front tree." Trees were used, instead of the wooden chests in Experiment 1, to maintain consistency with the outdoor setting portrayed in this virtual environment. As in the previous experiment, the virtual environment was amplified onto a $368 \times 282 \mathrm{~cm}$ white screen, $640 \times$ 480 pixels.

Procedure. Each participant was tested individually on one trial in the virtual reality lab. They were asked to fill in a brief questionnaire with the following instructions:

Your goal is to SURVIVE the attack of all the creatures. You will have to play the game for 5 minutes without being killed, and hopefully kill many creatures. You will use the mouse to shoot. In order to help you reach your goal, here are a few hints:

1 . The most dangerous creatures are the ones with a red gun, so try to shoot them first. However, they have a secret cloaking device, which means when they are a silver colour, they will not be affected by your bullets.

2. You only have a limited number of bullets, so use them wisely.

3. To shoot more effectively, use the green cross on the screen to identify your target. For example, if the green cross is directly on a creature, and you shoot, you will succeed in killing it.

4. Finally, be sure to take note of a big creature with wings that will appear at the beginning of the game. Although it cannot harm you, you will be asked about it later.

While you are playing the game, you will be asked a true or false question about the game. Give your answer out aloud. Thank you and enjoy your experience!

After reading the instructions, the participants began playing the game. There were two experimental conditions. In the first condition, the participants received a prerecorded linguistic prime specific to the assignment of the term front approximately 4 min into playing the game. Half of the participants in this condition had to respond truelfalse to the following linguistic prime: "During the game, the winged creature is in front of the other creatures." The response consistent with the ego-moving perspective of the prime was true. In order to counteract an affirmative response bias, the other half of the participants responded to the following linguistic prime: "During the game, the winged creature is behind other creatures." The response consistent with the ego-moving perspective of the prime was false.

In the second condition, the participants received a prerecorded question that did not refer to the spatial properties of any objects in the environment. Approximately 4 min into playing the game, the participants had to answer true or false to the following question: "During this game, there are a lot of creatures to shoot." Responses in this condition were not scored for correctness.

Once the participants had successfully survived the environment for an average of $6 \mathrm{~min}$, a prerecorded voice congratulated them for their survival skills and then presented them with the target task: "Shoot the front tree," using the game console. The experimenter noted which tree had been shot. At the end of the experiment, the participants received a short debriefing.

\section{Results and Discussion}

Of the 17 participants who received the linguistic prime, 2 of them did not answer the prime question in a manner consistent with the ego-moving perspective of the prime. These data were not used in the following analyses.

In the linguistic prime condition, all of the remaining 15 participants identified the winged creature as being located in front of the other creatures, regardless of the version of prime question. This means that all the participants who received the question "During the game, the winged creature is in front of the other creatures" responded true, and the participants who received the question "During the game, the winged creature is behind the other creatures" responded false.

Of the participants who received the nonspatial question $(n=13), 100 \%$ of them selected the tree that was closer to them, which is consistent with the object-moving perspective of the virtual environment. However, of the participants who received the linguistic prime, only $48 \%$ of them selected the tree that was closer to them. Instead, $52 \%$ of the participants in the first condition overcame the object-moving bias of the virtual environment and selected the farther tree as the front one, which is consistent with ego-moving perspective of the linguistic prime. A $2 \times 2$ chi-square analysis, conducted with a Yates's corrected chi-square because of low cell counts in the nonspatial question condition, confirmed a significant difference in the target tree in this environment $\left[\chi^{2}(1)=7.3\right.$, $p=.007]$.

Since the linguistic prime in this experiment was phrased in two different ways, this may have affected the participants' responses to the target task. For example, it is possible that the participants who received the prime question with the word front ("the winged creature is in front of the other creatures") would be more likely to consistently map the use of this word in the target task. In contrast, the participants who received the prime question with the word behind ("the winged creature is behind the other creatures") would be less sensitive to the label front in the target task and, thus, less likely to interpret front in the target task consistently with an object-moving schema. There is some support for this notion when the number of participants who received the priming question is examined. Out of the 7 participants who selected the closer tree as one in front, in accord with the object-moving perspective of the game (48\%), 5 of them received the prime question with the word behind. It is possible that because they had received a prime question that did not contain the word front, as in the target question, the priming was not as effective as it was for those who had received the prime question with the word front.

These results must be interpreted cautiously. In accord with previous studies (e.g., Boroditsky, 2000; Gentner \& Imai, 1992; McGlone \& Harding, 1998), the object-moving schema in the present study refers to a motion perspective in which the individual is stationary and objects move to- 
ward him or her. According to this motion perspective, an object furthest away from the individual is labeled as the front object. However, it is possible that in a system in which objects move away from, rather than toward, a stationary individual, the farther object, not the closer one, would be considered front. Since a virtual environment simulating this variation of the object-moving perspective was not developed (and has not been tested in previous related studies), we cannot exclude the possibility that the direction of motion may have contributed to the performance of the target task.

\section{GENERAL DISCUSSION}

In the present study, we investigated the roles of experience and language in disambiguating spatial terms. Through the use of a virtual environment, the participants were able to experience either an ego-moving or an objectmoving system. The findings indicated that experiencing a particular schema of motion influences how a target task is disambiguated. For the participants who received the nonspatial question, interacting with the virtual environment was powerful enough to significantly influence them to adopt the same spatial perspective when executing the ambiguous target task. For those who received a linguistic prime, they disambiguated the target task in a manner contrary to the virtual environment.

Previous research (both experimental and field studies) has shown that when primed with a particular motion perspective, individuals are more likely to disambiguate targets consistently with the prime (Boroditsky, 2000; Boroditsky \& Ramscar, 2002). For example, Boroditsky found that when presented with diagrams in a questionnaire format representing an ego-moving perspective, $63 \%$ of the participants disambiguated the target accordingly. When presented with object-moving diagrams, $67 \%$ were influenced by this spatial perspective when responding to the target. In the present study, $83 \%$ of the participants in the ego-moving virtual environment were affected by this perspective in interpreting the target task. In Experiment 2, $100 \%$ of the participants interpreted front in the target task consistently with the object-moving virtual environment. The percentage of participants in the present study who were affected by the spatial bias of the virtual environment is larger than in previous studies and suggests that the virtual task was effective in representing an embodied environment. This provides some empirical support for embodiment theories that suggest that physical experiences direct our understanding of concepts (e.g., Barsalou, 1999; Lakoff \& Johnson, 1999; see also Glenberg, 1997; Wilson, 2002). This finding is also consistent with research on reference frames, since the labeling of front in the ego-moving perspective of Experiment 1 is consistent with that in the encounter situation, where spatial terms are assigned relative to the viewer's perspective (Herskovits, 1986). In Experiment 2, the assignment of front in an object-moving perspective is in line with the accidental intrinsic scheme (Fillmore, 1982).
The finding that the linguistic bias affects the subsequent interpretation of spatial terms is also important. Previous studies have shown that linguistic primes can bolster the effect of embodied primes (e.g., Alloway et al., 1999). The present study extends these findings and indicates that the linguistic bias can influence an individual to "switch" spatial perspectives. This can be discussed in light of research on conceptual pacts, the idea that conversational partners will use the same lexical items in similar ways. Some researchers have found that speakers frequently engage in a language coordination process (Garrod \& Doherty, 1994). In a conversation, this means that language partners will use a communication exchange to build up a common context that can be used to interpret subsequent references. In particular, the notion of recency plays an important role in referencing an object (Brennan \& Clark, 1996; see also Garrod \& Anderson, 1987). People keep track of the most recent description used for an object and use this to formulate their current utterances. In the context of the present study, the participants who received the linguistic prime might have matched the use of the label front in the target task in such a way as to resemble the context in which they had first heard it applied.

Further support for this position was found in Experiment 2 when the participants who received the linguistic prime question were examined. Those who received the prime question with the word front were more likely to switch spatial perspectives and to apply the term front in the target task consistently with its use in the prime questions. In contrast, the majority of participants (5 out of 7) who received the prime question with the word behind were more influenced by the object-moving perspective of the virtual environment, rather than by the ego-moving perspective of the prime.

Although the findings from this study contribute to our understanding of the roles of experience and language in spatial perspective, there are some limitations. For example, the use of comparable layouts of the virtual environments for both Experiments 1 and 2 would have provided some additional validity that the motion effect was not influenced by the physical features in the virtual environment. In the present study, it was not possible to have used the ego-moving layout to simulate an object-moving perspective, since the ego-moving layout included buildings and other physical features that potentially would have obscured the viewing of objects moving toward an individual. Likewise, to use an object-moving layout to simulate an ego-moving perspective would have made the task too easy, since the layout was limited to a small area with minimal environmental features (only a door to a cave at the far end of the layout). However, this shortcoming could be addressed with a new design that matched the visual stimuli in the environment in both ego-moving and object-moving conditions. Furthermore, a within-subjects design would also strengthen the validity of the results from the present study.

The findings of the present study provide some support for the view that word meanings are not fixed but, 
rather, can be influenced by one's experience and linguistic context. The use of a virtual environment allows us to simulate an experience of motion and directly test the role of embodied experiences on spatial perspective. This extends previous research on spatial terms and provides an important contribution to the question of how both experience and linguistic context can influence our understanding of spatial perspective. The use of virtual environments to simulate motion when spatial perspectives are investigated is novel. Although the finding from the present study that experience can affect our application of spatial terms is encouraging, further work needs to be carried out in order to replicate these findings and validate the roles of language and experience in directing spatial perspective.

\section{REFERENCES}

Alloway, T. P., Ramscar, M., \& Corley, M. (1999). Verbal and embodied priming in schema mapping tasks. In M. Hahn \& S. C. Stoness (Eds.), Proceedings of the Twenty-First Annual Conference of the Cognitive Science Society (pp. 13-18). Hillsdale, NJ: Erlbaum.

Barsalou, L. (1999). Perceptual symbol systems. Behavioral \& Brain Sciences, 22, 577-609.

Boroditsky, L. (2000). Metaphoric structuring: Understanding time through spatial metaphors. Cognition, 75, 1-28.

Boroditsky, L., \& RAMSCAR, M. (2002). The roles of body and mind in abstract thought. Psychological Science, 13, 185-188.

Brennan, S. E., \& Clark, H. H. (1996). Conceptual pacts and lexical choice in conversation. Journal of Experimental Psychology: Learning, Memory, \& Cognition, 22, 1482-1493.

Carlson-Radvansky, L. A., \& Logan, G. D. (1997). The influence of reference frame selection on spatial template construction. Journal of Memory \& Language, 37, 411-437.

Couclelis, H., Golledge, R., Gale, N., \& Tobler, W. (1987). Exploring the anchor-point hypothesis of spatial cognition. Journal of Environmental Psychology, 7, 99-122.

Fillmore, C. J. (1971). The Santa Cruz lectures on deixis. Bloomington: Indiana University Linguistics Club.

Fillmore, C. J. (1982). Towards a descriptive framework for spatial deixis. In R. Jarvella \& W. Klein (Eds.), Speech, place and action: Studies in deixis and related topics (pp. 31-59). New York: Wiley.

Garrod, S., \& Anderson, A. (1987). Saying what you mean in dialogue: A study in conceptual and semantic co-ordination. Cognition, 27, 181-218.

Garrod, S., \& Doherty, G. (1994). Conversation, co-ordination and convention: An empirical investigation of how groups establish linguistic conventions. Cognition, 53, 181-215.

Gentner, D., \& Imai, M. (1992). Is the future always ahead? Evidence for system-mappings in understanding space-time metaphors. In Proceedings of the Fourteenth Annual Conference of the Cognitive Science Society (pp. 510-515). Hillsdale, NJ: Erlbaum.

Glenberg, A. M. (1997). What memory is for. Behavioral \& Brain Sciences, 20, 1-19.

Harris, L. J., \& STrommen, E. A. (1972). The role of front-back features in children's "front," "back," and "beside" placements. Merrill-Palmer Quarterly, 18, 259-271.

Herskovits, A. (1986). Language and spatial cognition: An interdisciplinary study of the prepositions of English. Cambridge: Cambridge University Press.

LAKOFF, G., \& Johnson, M. (1999). Philosophy in the flesh: The embodied mind and its challenge to Western thought. New York: HarperCollins.

Levinson, S. C. (1996). Frames of reference and Molyneux's question: Crosslinguistic evidence. In P. Bloom, M. A. Peterson, L. Nadel, \& M. Garrett (Eds.), Language and space (pp. 109-169). Cambridge, MA: MIT Press.

McGlone, M., \& Harding, J. (1998). Back (or forward?) to the future: The role of perspective in temporal language comprehension. Journal of Experimental Psychology, 24, 1211-1223.

Miller, G. A., \& Johnson-Laird, P. N. (1976). Language and perception. Cambridge, MA: Harvard University Press.

Sun, H.-J., Chan, G. S. W., \& Campos, J. L. (2004). Active navigation and orientation-free spatial representations. Memory \& Cognition, 32, 51-71.

Waller, D., Loomis, J. M., \& Haun, D. B. M. (2004). Body-based senses enhance knowledge of directions in large-scale environments. Psychonomic Bulletin \& Review, 11, 157-163.

Wilson, M. (2002). Six views of embodied cognition. Psychonomic Bulletin \& Review, 9, 625-636.

(Manuscript received May 11, 2004; revision accepted for publication March 11, 2005.) 